\title{
Experience Report: \\ Design and Implementation of a Component-Based Protection Architecture for ASP.NET Web Services
} Konstantin Beznosov

Laboratory for Education and Research in Secure Systems Engineering (LERSSE) Electrical and Computer Engineering University of British Columbia 


\section{How ASP.NET Web Services Work}

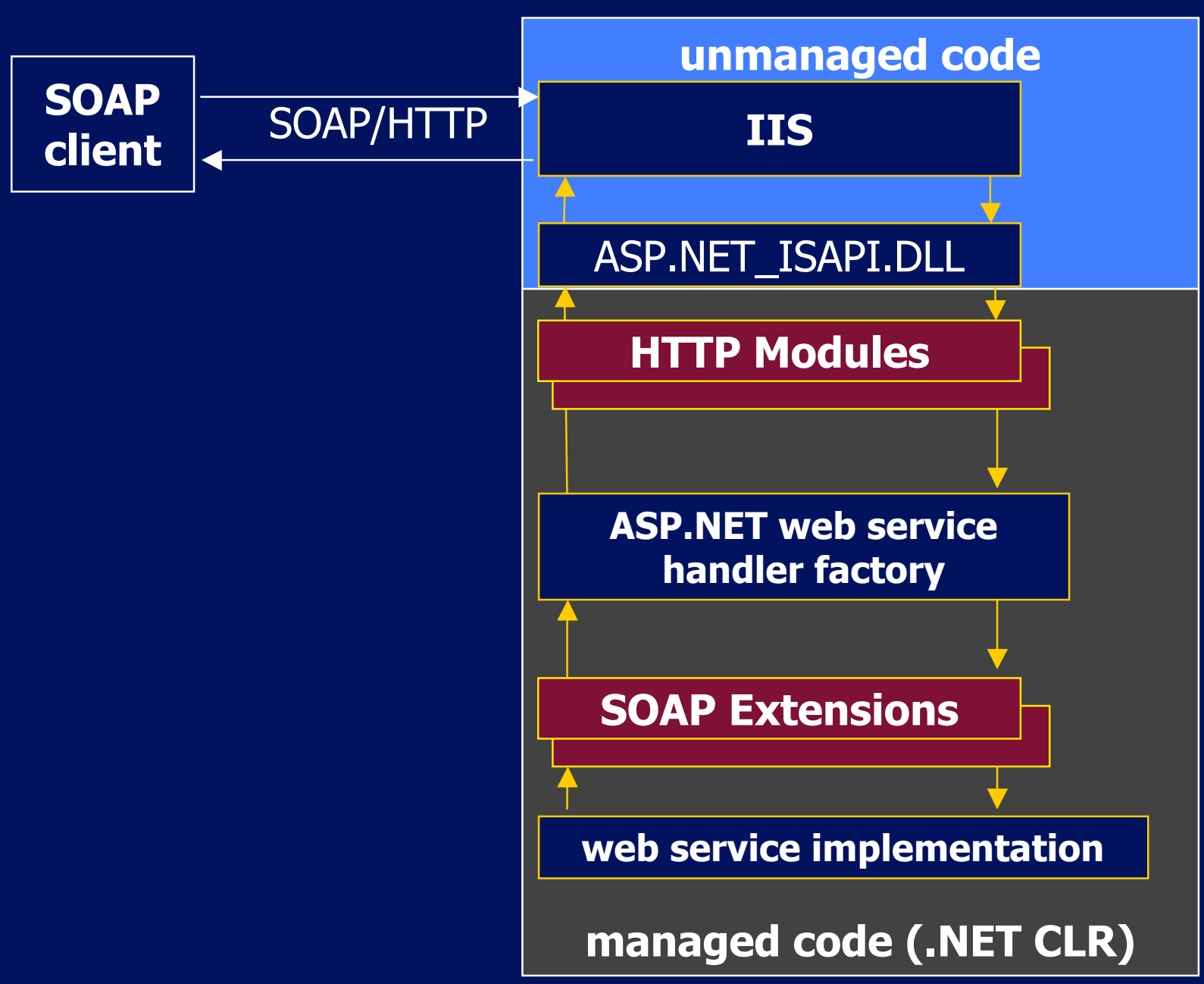




\section{ASP.NET Web Services Security}

Disclaimer: Biased, qualitative, unsupported comparison

\begin{tabular}{|l|r|r|}
\hline & Out-of-the-box & $\begin{array}{c}\text { Reported } \\
\text { Architecture }\end{array}$ \\
\hline granular & $* * *$ & $* * * * *$ \\
\hline scalable & $* * * *$ & $* * * * *$ \\
\hline extensible & $*$ & $* * * * *$ \\
\hline reusable & $* * *$ & $* * * * *$ \\
\hline
\end{tabular}




\section{Outline}

- System architecture

- Examples

- Lessons learned

- Summary 


\section{Reported System}

\section{What is it?}

Component-based

Authentication and Authorization (A\&A) architecture for ASP.NET Web services

\section{Key features}

Less effort to integrate into enterprise security

1. More granularity and scalability: scalable and finegrained configuration of machine-wide A\&A functions

2. More extensibility: easy to add new A\&A logic

3. Better reusability: A\&A components can be combined 


\section{Separation of Enforcements \& Decisions}

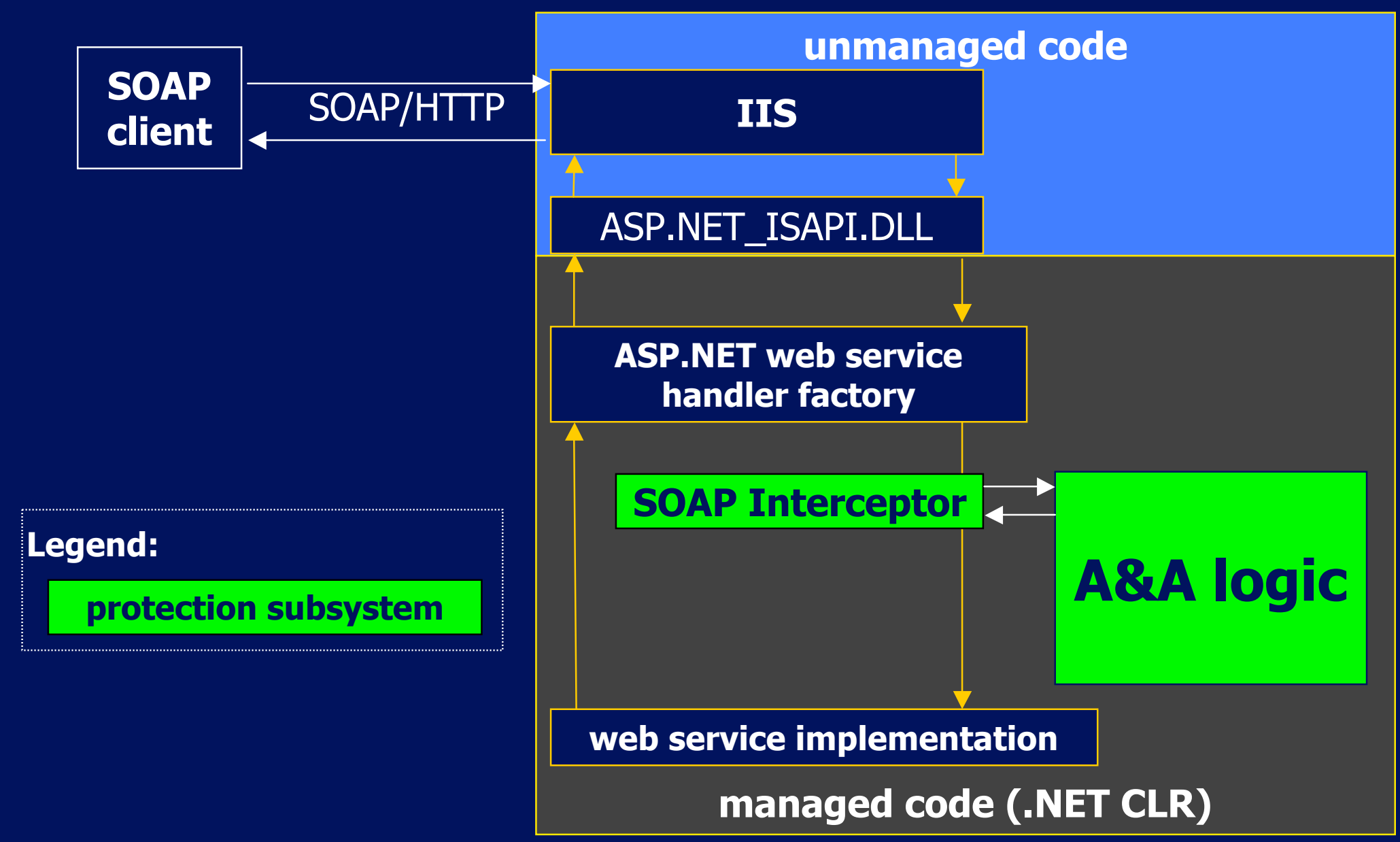

Interceptor enforces, "A\&A logic" decides 


\section{Component Framework for A\&A Logic}

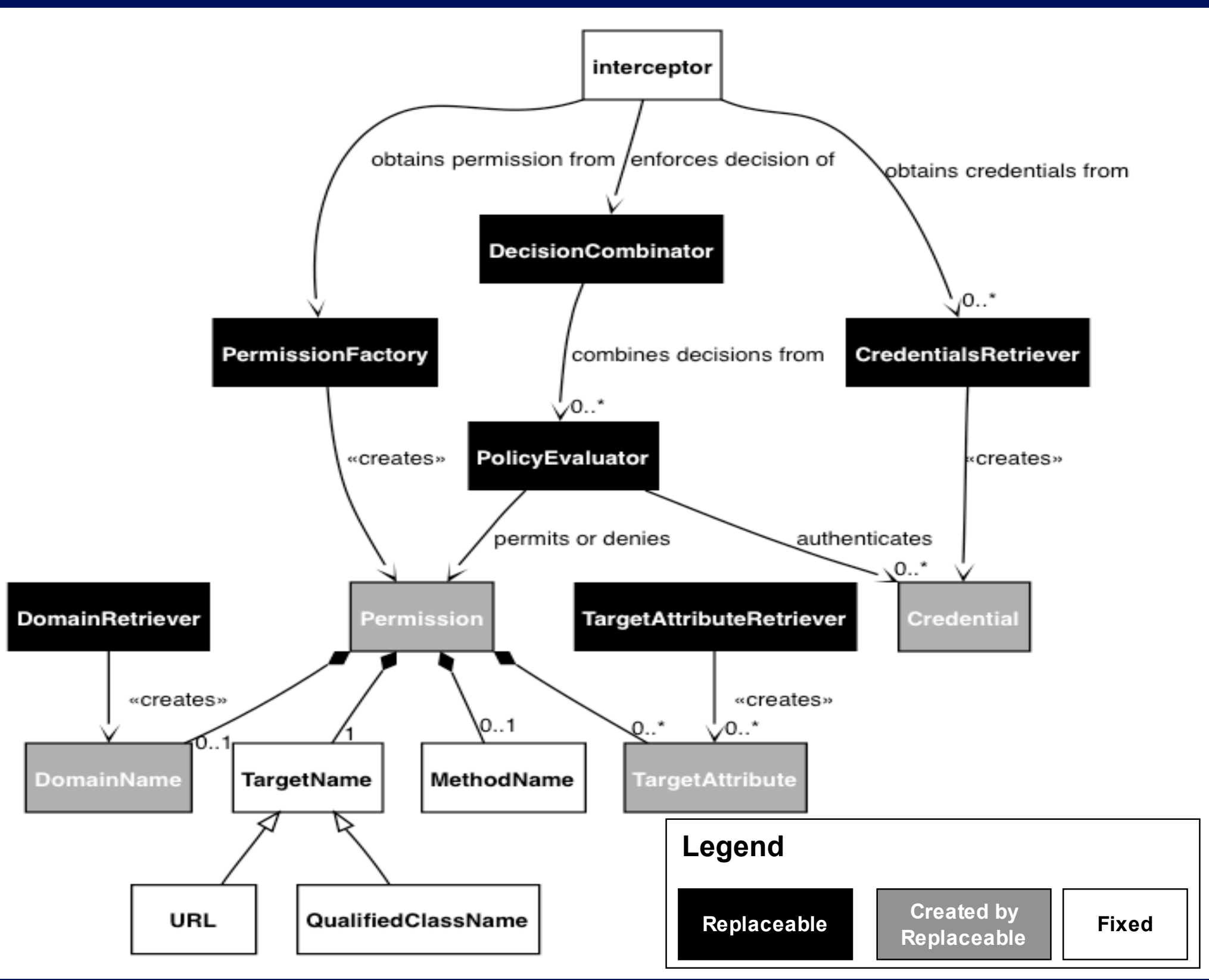




\section{Permission Examples}

\begin{tabular}{|l|l|}
\hline Permission Example & Explanation \\
\hline http://foobank.com/bar.asmx & Only the URL is used \\
\hline com.foobank.ws.Sbar/m1 & Class and method names \\
\hline D1/com.foobank.ws.Sbar/m1 & Same but in domain "D1" \\
\hline com.foobank.ws.Sbar/owner=smith & Class name and attribute \\
\hline D1/com.foobank.ws.Sbar/owner=smith/m1 & Domain / class / attribute / method \\
& \\
\hline
\end{tabular}




\section{Call Sequence}

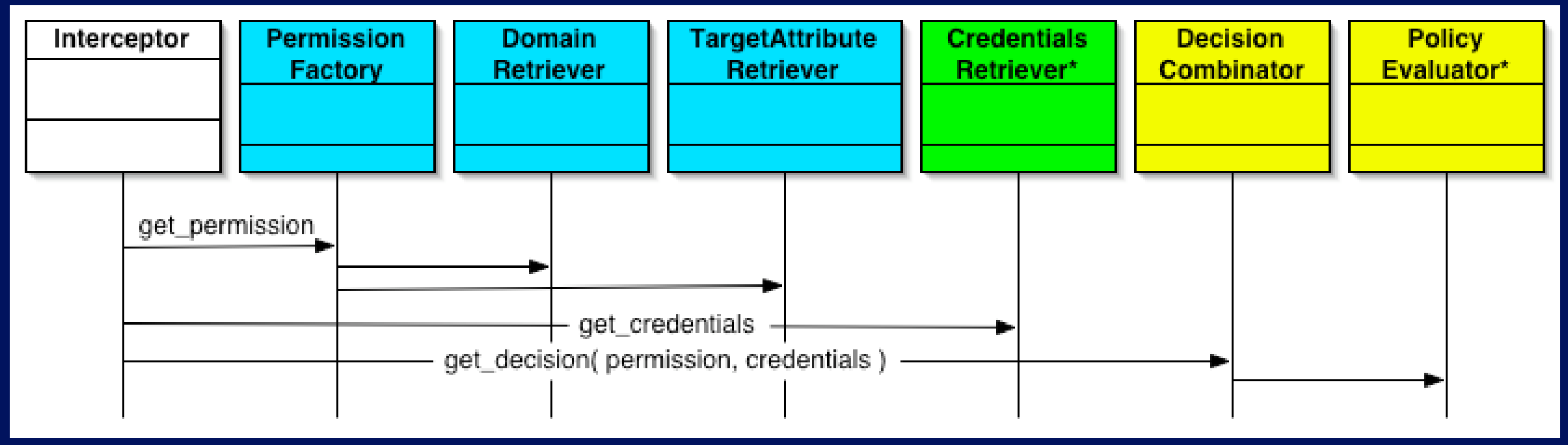




\section{UBC}

THE UNIVERSITY OF BRITISH C OLUMBIA

\section{Example 1}

University Course Web Service 


\section{University Course Web Service Policy}

1. Anyone can lookup course descriptions.

2. All users should authenticate using HTTP-BA.

3. Registration clerks can list students registered for the course and (un)register students.

4. The course instructor can list registered students as well as manage course content.

5. Registered for the course students can download assignments and course material, as well as submit assignments. 


\section{Policy Engine Assembly for Example 1}

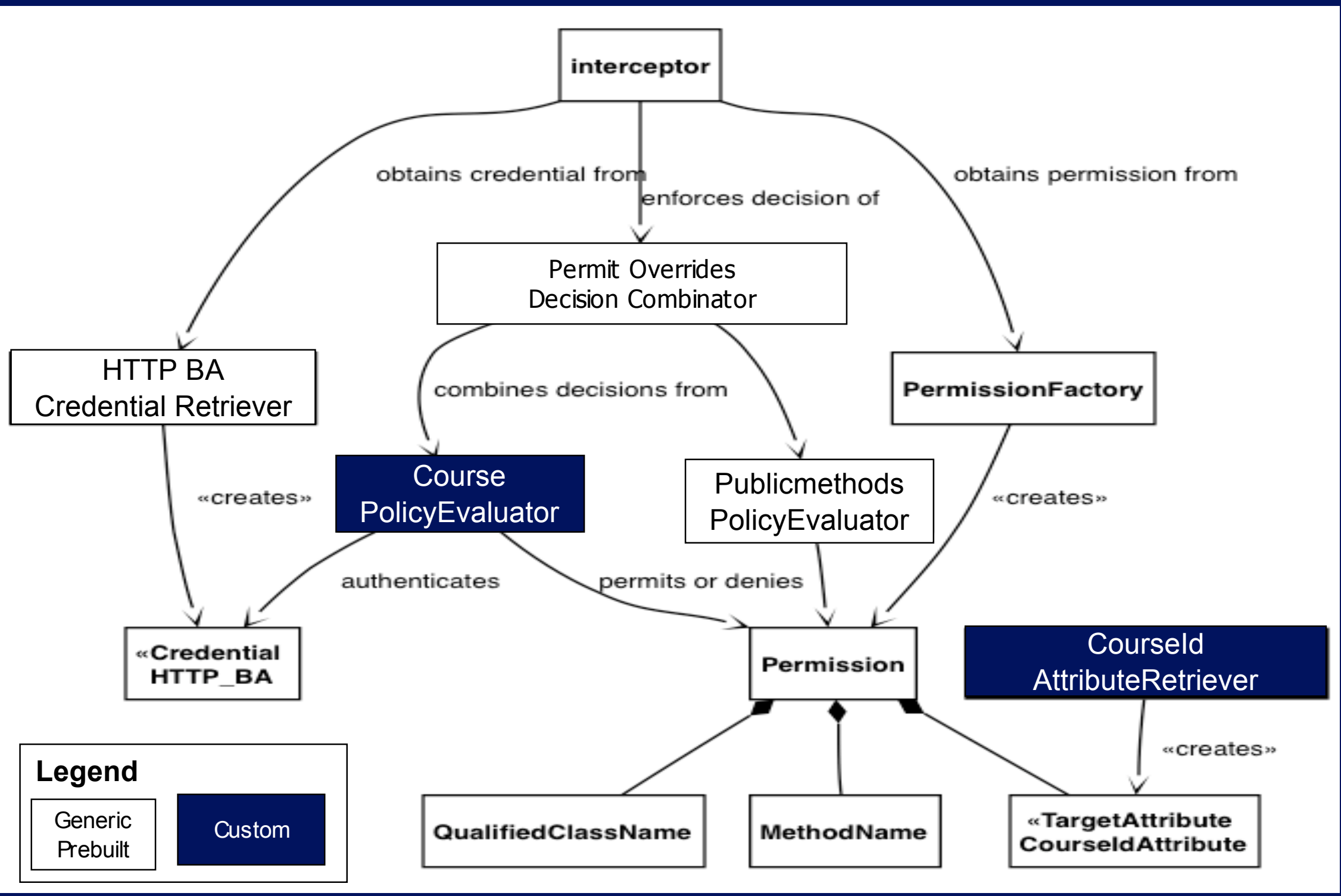




\title{
UBC
}

THE UNIVERSITY OF B RITISH C OLUMBIA

\section{Example 2}

\author{
Human Resources Web Service \\ for an International Organization
}




\section{HR Web Service Policy}

1. Only users within the company's intranet or those who access the service over SSL and have valid X.509 certificates issued by the company should access.

2. Anybody in the company can look up any employee and get essential information about her/him.

3. HR employees can modify contact information and review salary information of any employee from the same division.

4. HR managers can modify any information about the employees of the same division. 


\section{Policy Engine Assembly for Example 2}

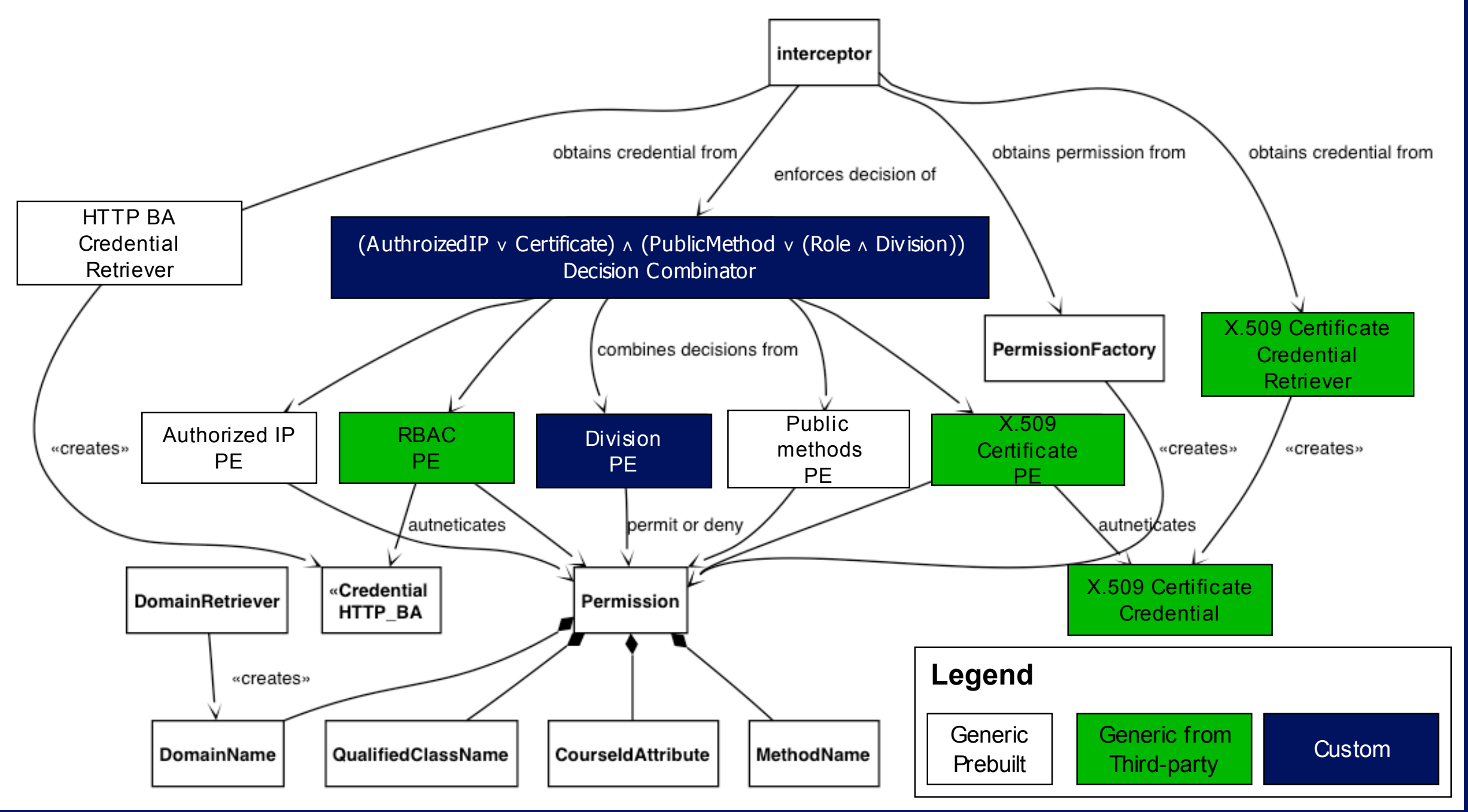




\section{Expected Lessons Learned}

- It's possible to design security decision logic as components

- reusable from policy to policy

- composable to support different policies

- replaceable to allow new policies

- ASP.NET container is suitable for extensions (in the form of components)

- effective design required deep understanding of access control, Web services, and (ASP).NET

- effective configuration (packaging) crucial

- embracing (not ignoring or suppressing) ASP.NET idiosyncrasies lead to the success 


\section{Expected Lessons Learned}

- It's possible to design security decision logic as components

- reusable from policy to policy

- composable to support different policies

- replaceable to allow new policies

- ASP.NET container is suitable for extensions (in the form of components)

- effective design required deep understanding of access control, Web services, and (ASP).NET

- effective configuration (packaging) crucial

- embracing (not ignoring or suppressing) ASP.NET idiosyncrasies lead to the success 


\section{Unexpected Lessons Learned}

- customers did not care that much about standard compliance \& interoperability

- hard to interpret very flexible WS-Security spec

- switching to XP-like User Stories too shocking

- avoid showing all the capabilities/flexibility

- unscalable life-cycle of interceptors

- SOAP interceptor intercepts only SOAP messages (duh!) 


\section{Summary}

- experience report about designing and implementing protection framework for ASP.NET Web services

- (un)expected lessons learned

- $\mathrm{CB}$ authentication and authorization mechanisms

- feasable

- evolve with policies

- details

- in the paper

- http://konstantin.beznosov.net 